\title{
Breaking the diffraction limit with dynamic saturation optical microscopy
}

\author{
Jörg Enderlein ${ }^{\text {a) }}$ \\ Institute for Biological Information Processing 1, Forschungszentrum Jülich, D-52425 Jülich, Germany
}

(Received 2 June 2005; accepted 20 July 2005; published online 23 August 2005)

\begin{abstract}
A scheme of fluorescence microscopy is proposed allowing the breaking of the diffraction limit of optical microscopy by a factor of four to five. It relies on fast temporal measurements of the fluorescence decay after sudden switch-on of the light excitation. The observed temporal dynamics of the fluorescence signal can be converted into information about the spatial distribution of fluorophores within the exciting laser focus. The proposed scheme is technically simple, allows resolution enhancement in three dimensions, and will be robust with respect to small optical aberrations as caused by refractive index variations in real samples. (C) 2005 American Institute of Physics. [DOI: 10.1063/1.2034116]
\end{abstract}

The primary limitation of the classical light microscope is its finite spatial resolution, which is limited by the diffraction of light to about $200 \mathrm{~nm}$ in the lateral direction (image plane) and about $600 \mathrm{~nm}$ along the axial direction (optical axis). In recent years, several methods had been developed aiming at the extension of the resolution limit of the classical light microscope as set by the diffraction of light. ${ }^{1-4}$ All these methods are still restricted in their spatial resolution by the diffraction of light, principally not breaking the resolution limit as set by diffraction; for a more detailed overview see Ref. 5. Since the inception of nonlinear optics a few decades ago, ${ }^{6}$ it has been clear that a nonlinear relationship between the applied intensity and the measured fluorescence signal would - at least in principle-expand the resolution capabilities of a focusing ("far-field") light microscope. However, only in the early 1990s concrete physical concepts appeared for breaking the diffraction barrier of resolution, resulting in a whole family of concepts that utilize reversible saturable optical (fluorescence) transitions which was termed by Hell et al. RESOLFT. ${ }^{7,8}$

Consider the particular case of fluorescing molecules having a ground state $S_{0}$, excited state $S_{1}$, and triplet state $T_{1}$. It is assumed that the transition rate from the excited to the ground state (inverse of the fluorescence lifetime $\tau$ ) is much faster than the intersystem-crossing rate $k_{\text {isc }}$.

When a fluorescing sample is illuminated with some spatially varying intensity distribution, the position dependent probability $s(\mathbf{r})$ to find the molecules either in the state $S_{0}$ or $S_{1}$ is found by solving the kinetic equation

$$
\frac{d s(\mathbf{r}, t)}{d t}=-\frac{\tau k_{\mathrm{ex}}(\mathbf{r}) k_{\mathrm{isc}}}{1+\tau k_{\mathrm{ex}}(\mathbf{r})} s(\mathbf{r}, t)+k_{\mathrm{ph}}[1-s(\mathbf{r}, t)],
$$

where $k_{\mathrm{ex}}(\mathbf{r})$ is the position dependent excitation rate which is directly proportional to the illuminations intensity distribution, and $k_{\mathrm{ph}}$ is the inverse triplet state lifetime (phosphorescence rate). If the laser light is switched on suddenly, so that at time $t=0$ all molecules are in the ground state $S_{0}$, i.e., $s(\mathbf{r}, t=0)=1$, the explicit solution of this equation is given by

${ }^{a)}$ Electronic mail: j.enderlein@fz-juelich.de

$$
\begin{aligned}
s(\mathbf{r}, t)= & \frac{k_{\mathrm{ph}}}{k_{\mathrm{ph}}+\tau k_{\mathrm{isc}} f(\mathbf{r})}+\frac{\tau k_{\mathrm{isc}} f(\mathbf{r})}{k_{\mathrm{ph}}+\tau k_{\mathrm{isc}} f(\mathbf{r})} \\
& \times \exp \left\{-\left[k_{\mathrm{ph}}+\tau k_{\mathrm{isc}} f(\mathbf{r})\right] t\right\},
\end{aligned}
$$

where the abbreviation

$$
f(\mathbf{r})=\frac{k_{\mathrm{ex}}(\mathbf{r})}{1+\tau k_{\mathrm{ex}}(\mathbf{r})}
$$

was used. For most dyes, the triplet state lifetime $k_{\mathrm{ph}}^{-1}$ is of the order of a few microseconds, so that static equilibrium in Eq. (2) is reached on that time scale, after which the position dependent fluorescence signal $F(\mathbf{r})$ is given by

$$
F(\mathbf{r})=f(\mathbf{r}) s(\mathbf{r}, t \rightarrow \infty)=\frac{k_{\mathrm{ph}} f(\mathbf{r})}{k_{\mathrm{ph}}+\tau k_{\mathrm{isc}} f(\mathbf{r})} .
$$

The core idea of RESOLFT is that, due to the highly nonlinear relation between the exciting illumination profile $I(\mathbf{r})$ and the resulting fluorescence profile $F(\mathbf{r})$, one can generate arbitrarily steep spatial modulations in $F(\mathbf{r})$, depending only on the intensity modulation amplitude of $I(\mathbf{r})$, with much higher frequencies in Fourier space than originally contained in the generating distribution $I(\mathbf{r})$. This directly translates into correspondingly higher spatial resolution in an imageforming process when scanning a sample with such a modulated intensity distribution $I(\mathbf{r})$. For the special case of the singlet/triplet state transition considered above, the resulting method of image resolution enhancement is a particular variant of the so-called saturated patterned excitation microscopy (SPEM) as proposed by Heintzmann et al. ${ }^{9,10}$ A more sophisticated realization of RESOLFT is stimulated emission depletion (STED) microscopy, developed by Hell et al. ${ }^{11-13}$

Here, I propose a different approach for increasing the spatial resolution in imaging beyond the limit of light diffraction by explicitly using the temporal dynamics of fluorescence. For simplicity, consider a localized excitation intensity profile which is suddenly switched on at time zero. At every point, the excited fluorescence will be proportional to $f(\mathbf{r})$ times $s(\mathbf{r}, t)$, where the temporal dynamics of $s(\mathbf{r}, t)$ is a superposition of an exponential decay plus a constant offset (the infinite time limit). At any position, the decay rate is directly proportional to the excitation intensity, see Eq. (2). The rapidity with which the fluorescence signal reaches equilibrium directly reflects the local excitation intensity. This is exemplified in Fig. 1: Any point along a one-dimensional 

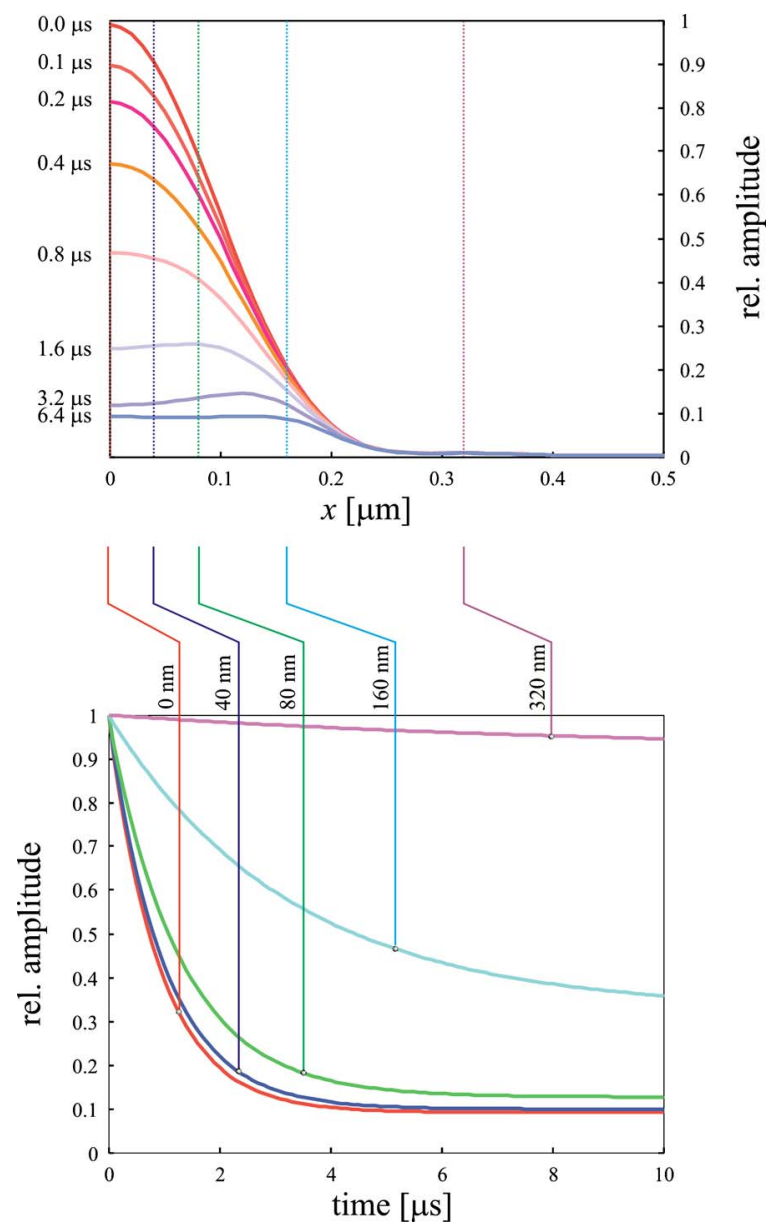

FIG. 1. (Color online) Converting temporal into spatial information: The upper graph shows the temporal evolution of the fluorescence intensity profile after switching on a Gaussian laser beam illuminating a homogeneous distribution of fluorophores. The photophysical parameters used are: fluorescence lifetime $1 \mathrm{~ns}$, triplet state lifetime $10 \mu \mathrm{s}$, intersystem crossing rate $10^{8} / \mathrm{s}$. Peak excitation rate (at zero position) is assumed to be $10 \mathrm{MHz}$. The bottom graph shows the fluorescence signal decay as would be measured at the different indicated positions: The higher the excitation intensity, the faster the molecules are pumped into the triplet state and the faster the fluorescence signal approaches its long-time equilibrium level.

Gaussian excitation intensity profile corresponds to a different decay rate of the fluorescence signal. Consequently, the temporal information contained in the dynamics of the fluorescence signal is directly related to the spatial information as given by the spatial modulation of the excitation intensity. For the sake of simplicity, let us assume that all the fluorescence generated by the localized excitation intensity distribution is collected by some detection optics with uniform collection efficiency. Then for any center position $\mathbf{r}$ of the excitation profile, the detected fluorescence signal is given by the integral

$$
I(\mathbf{r}, t)=\int d \mathbf{r}^{\prime} s\left(\mathbf{r}-\mathbf{r}^{\prime}, t\right) f\left(\mathbf{r}-\mathbf{r}^{\prime}\right) c\left(\mathbf{r}^{\prime}\right)
$$

where $c\left(\mathbf{r}^{\prime}\right)$ describes the distribution of fluorophores in the sample. Using Eq. (2), this expression can be rewritten into a different form,

$$
I(\mathbf{r}, t)=I_{\mathrm{eq}}(\mathbf{r})+\int d \Gamma w(\Gamma) \exp (-\Gamma t)
$$

where $I_{\mathrm{eq}}(\mathbf{r})$ denotes the fluorescence signal which is generated under equilibrium conditions, and $w(\Gamma)$ is a weight function defined by bution with a narrower central peak for the prize of much
Downloaded 21 Dec 2006 to 134.94.122.39. Redistribution subject to AlP license or copyright, see http://apl.aip.org/apl/copyright.jsp

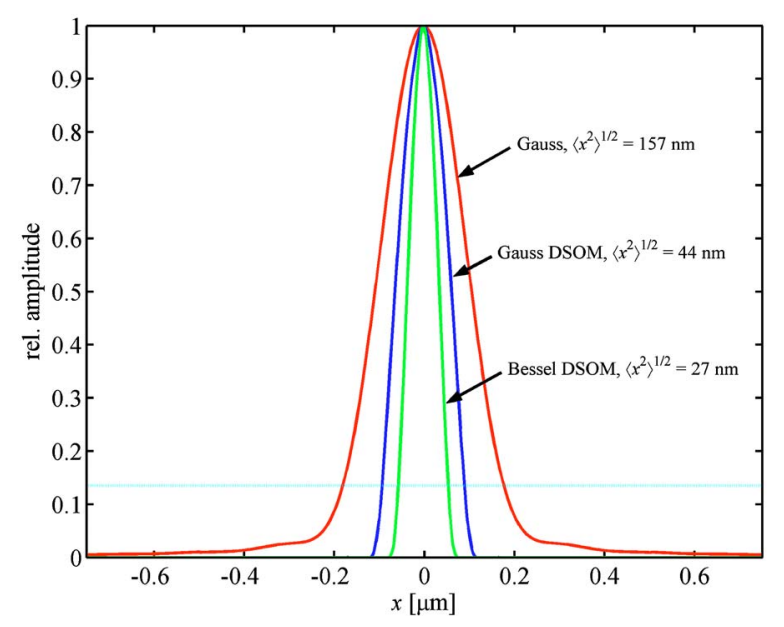

FIG. 2. (Color online) Point spread function obtained when scanning an infinitely narrow line distribution of fluorescent molecules. Shown are the resulting signals for a diffraction limited focus in the limit of no optical saturation, the same focus with DOSM, and a Bessel focus with DOSM.

$$
\begin{aligned}
w(\Gamma)= & \left(1-\frac{k_{\mathrm{ph}}}{\Gamma}\right) \int d \mathbf{r}^{\prime} c\left(\mathbf{r}+\mathbf{r}^{\prime}\right) f\left(\mathbf{r}^{\prime}\right) \\
& \times \delta\left[k_{\mathrm{ph}}+\tau k_{\mathrm{isc}} f\left(\mathbf{r}^{\prime}\right)-\Gamma\right] .
\end{aligned}
$$

The integration extends from $\Gamma_{\min }=k_{\mathrm{ph}}$ up to $\Gamma_{\max }=k_{\mathrm{ph}}$ $+\tau k_{\text {isc }} \max [f(\mathbf{r})]$. All the RESOLFT methods that are described in the cited literature use solely the equilibrium part $I_{\text {eq }}(\mathbf{r})$,

$$
I_{\mathrm{eq}}(\mathbf{r})=\int d \mathbf{r} \frac{k_{\mathrm{ph}} f\left(\mathbf{r}-\mathbf{r}^{\prime}\right)}{k_{\mathrm{ph}}+\tau k_{\mathrm{isc}} f\left(\mathbf{r}-\mathbf{r}^{\prime}\right)} c\left(\mathbf{r}^{\prime}\right),
$$

and completely ignore the time-dependent part. To our knowledge, the only work that has ever discussed the use of temporal information in the fluorescence signal for improving imaging resolution is Ref. 14, but within a completely different context of a double color excitation employing a hypothetical reversible $S_{1} \leftrightarrow X_{n}$ transition. Close inspection of Eqs. (5) and (6) shows that every decay time $\Gamma$ of the signal at a given point $\mathbf{r}$ samples the fluorescence within infinitely thin regions around $\mathbf{r}$ which are defined by the above $\delta$-function. The important point is that a certain range $\Delta \Gamma$ of decay rates defines a finite spatial region that can be much smaller than the optical wavelength.

This is the physical principle behind the concept of enhancing the spatial resolution of imaging which will be termed dynamic saturation optical microscopy (DSOM).

As a numerical example consider a fluorescence lifetime of $\tau=1 \mathrm{~ns}$, intersystem crossing quantum yield of $10 \%$, i.e., $\tau k_{\text {isc }}=0.1$, triplet state lifetime $k_{\mathrm{ph}}^{-1}=10 \mu \mathrm{s}$, and a maximum excitation rate of $f_{\max }=10 \mathrm{MHz}$ (at focus center position), which is easily achieved with moderate excitation power within a diffraction limited focus. Thus, we assume excitation by a diffraction limited laser focus which is produced by an oil-immersion objective with 1.4 numerical aperture (NA). Calculation of the excitation intensity distribution was done following the theory of Wolf and Richards for high-NA optical systems. ${ }^{15}$ The resulting standard deviation of the excitation light distribution along one dimension is $157 \mathrm{~nm}$. As a different excitation mode, a so-called Bessel beam generated by focusing a ring of radially polarized light through the same objective was also considered, generating a light distribution with a narrower central peak for the prize of much 

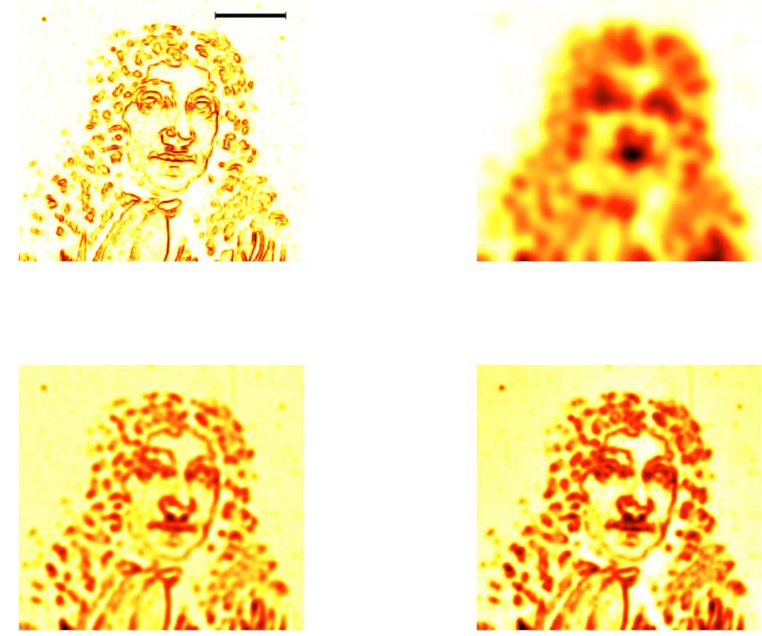

FIG. 3. (Color online) Demonstration of DSOM performance on a richly structured sample. Top left panel shows the assumed fluorophore distribution (graph of a portrait of microscopy pioneer Antonin van Leuwenhoek), and the length of the black bar is $1 \mu \mathrm{m}$. The top right panel shows an image as obtained when scanning with the diffraction limited focus in the limit of no optical saturation, the bottom left when using DSOM, and the bottom right panel when using DSOM with a Bessel beam focus.

elevated side peaks. ${ }^{16,17}$ Without restriction of generality, we will focus here on imaging in two dimensions. For measuring the temporal decay of the fluorescence signal on a microsecond time scale due to the transition of the molecules from the singlet to the triplet state, scanning and excitation of the sample has to be performed in a discontinuous way: The laser focus is positioned at consecutive points in the sample, and only after positioning, the excitation light is switched on for a few microseconds and then switched off again. Before measuring the signal at the next position, one has to wait long enough that all molecules have time to return from the triplet to the singlet ground state. Thus, for the above numbers, a reasonable measurement mode will be a $10 \mu$ s ontime of the excitation light with $\approx 100 \mu$ s off-times in between. Spatial scanning steps have also to be chosen small enough to match the spatial resolution that one desires to achieve. For the numerical example studied here, a step width of $2.5 \mathrm{~nm}$ was chosen. Each recorded curve is fitted by a discrete set of four exponentially decaying intensity profiles with 1, 2, 4 and $8 \mu$ s decay times using a simple but efficient linear least square procedure. Although it does not guarantee non-negativeness of the decay component amplitudes, it worked sufficiently well for extracting the amplitude of the fastest decay component $(1 \mu \mathrm{s})$. As a first test, a twodimensional scan of an infinitely thin fluorescing line was simulated. Figure 2 shows a comparison between the signal as directly observed when scanning with the diffraction limited focus in the limit of no optical saturation, the signal as observed when using the same profile for the DSOM procedure, and the signal when using the Bessel beam together with DSOM. For the DSOM profiles in Fig. 2, the fitted amplitude of the shortest considered decay component is plotted. The case of the Bessel beam-DSOM shows important and beneficial side effect of DSOM: Because the fastest decay in the fluorescence signal is generated by regions of highest excitation intensity, the significant but much lower amplitude side peaks in the Bessel beam distribution are completely suppressed in the resulting DSOM signal. Thus,
DSOM could be an ideal supplement for techniques such as 4Pi microscopy, where a narrow central light distribution along the optical axis is accompanied by smaller but nonnegligible side peaks that have conventionally to be eliminated by sophisticated image restoration procedures. ${ }^{18}$ For a more intuitive visualization of the performance of the DSOM, we will use a microscopic copy of an image of the microscopy pioneer Antonin van Leuwenhoek. Figure 3 shows four panels: The supposed distribution of fluorescent molecules (the original Leuwenhoek picture); the resulting image when using conventional scanning and detection with the diffraction limited excitation focus; the image using the same focus but with DSOM; and the image using a Bessel beam focus with DSOM. The increased spatial resolution in imaging produced by DSOM is obvious. The gain in DSOM resolution when using a Bessel beam instead of a Gaussian beam can be seen when looking at finer image details such as the curls of the hair. The background in the DSOM images is caused by the rather pure performance of the simple nonlinear least-square algorithm. Experimental realizations may use more sophisticated algorithms such as non-negative least square analysis.

Several generalizations and modifications of the DSOM concept are feasible. Instead of using the singlet-triplet transition dynamics, one can directly employ the saturation of the ground to first excited single state transition. For such an implementation, fluorescing molecules or particles with long fluorescence lifetimes from several dozen nanoseconds up to microseconds will be preferable, such as quantum dots, diamond color centers, or rare earth fluorescent dyes. Besides excited or triplet states, any other optically saturable transition can also be employed for realizing DSOM. Furthermore, the concept of DSOM is not restricted to scanning microscopy. Using spatially modulated excitation and a fast gated camera for imaging, DSOM could be used for enhancing the spatial resolution in whole-field imaging.

The author thanks Thomas Ruckstuhl and Ingo Gregor for many inspiring discussions. The author is grateful to Benjamin Kaupp and Eike Stedefeldt for their general support of my work.

${ }^{1}$ S. W. Hell and H. K. Stelzer, J. Opt. Soc. Am. A 9, 2159 (1992).

${ }^{2}$ M. G. L. Gustafsson, D. A. Agard, and J. W. Sedat, J. Microsc. 195, 10 (1999).

${ }^{3}$ M. G. L. Gustafsson, J. Microsc. 198, 82 (2000).

${ }^{4}$ W. Denk, J. H. Strickler, and W. W. Webb, Science 248, 73 (1990).

${ }^{5}$ M. G. L. Gustafsson, Curr. Opin. Struct. Biol. 9, 627 (1999).

${ }^{6}$ N. Bloembergen, Nonlinear Optics (Benjamin, Amsterdam, 1965).

${ }^{7}$ S. W. Hell, Nat. Biotechnol. 21, 1347 (2003).

${ }^{8}$ S. W. Hell, M. Dyba, and S. Jakobs, Curr. Opin. Neurobiol. 14, 1 (2004).

${ }^{9}$ R. Heintzmann, T. M. Jovin, and C. Cremer, J. Opt. Soc. Am. A 19, 1599 (2002).

${ }^{10}$ R. Heintzmann, Micron 34, 283 (2003).

${ }^{11}$ S. W. Hell and J. Wichmann, Opt. Lett. 19, 1 (1994).

${ }^{12}$ S. W. Hell and M. Kroug, Appl. Phys. B: Lasers Opt. 60, 495 (1995).

${ }^{13} \mathrm{~S}$. W. Hell, in Topics in Fluorescence Spectroscopy, 5th ed., edited by J. R. Lakowicz (Plenum, New York, 1997), pp. 361-422.

${ }^{14}$ A. Schönle and S. W. Hell, Eur. Phys. J. D 6, 283 (1999).

${ }^{15}$ B. Richards and E. Wolf, Proc. R. Soc. London, Ser. A 253, 358 (1959).

${ }^{16}$ S. Quabis, R. Dorn, M. Eberler, O. Glöckl, and G. Leuchs, Appl. Phys. B: Lasers Opt. 72, 109 (2001).

${ }^{17}$ M. A. Lieb and A. J. Meixner, Opt. Express 8, 458 (2001).

${ }^{18}$ M. Schrader, S. W. Hell, and H. T. M. van der Voort, J. Appl. Phys. 84, 4033 (1998). 